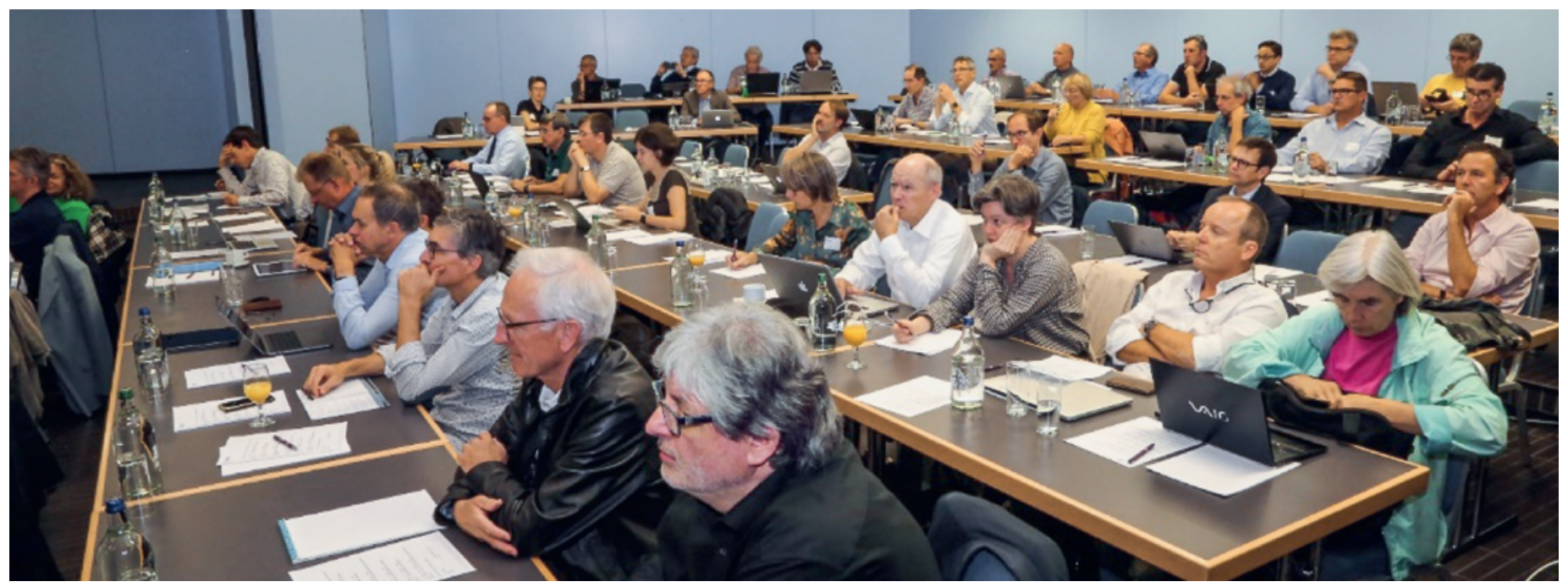

Les exposés ont suscité un vif intérêt auprès des participants à la Journée des délégués tarifaires.

\title{
Dernière ligne droite pour la révision tarifaire?
}

\author{
Patrick Müllera ${ }^{a}$, Thomas Kessler ${ }^{b}$, Susanne Christen ${ }^{c}$, Christian Oeschger ${ }^{d}$ \\ ${ }^{a} \mathrm{FMH}$, chef de division, division Médecine et tarifs ambulatoires; ${ }^{b} \mathrm{FMH}$, expert, division Médecine et tarifs ambulatoires; ${ }^{\mathrm{c}} \mathrm{Dresse}, \mathrm{FMH}$, experte, \\ cheffe suppléante, division Médecine et tarifs ambulatoires; ${ }^{d}$ FMH, expert, division Médecine et tarifs ambulatoires
}

Sous le titre "Dernière ligne droite pour la révision tarifaire?», la deuxième Journée des délégués tarifaires de l'année qui s'est tenue le 27 septembre 2018 à Berne a réuni plus de 100 participants, un nombre élevé qui semble se stabiliser.

\section{Dimension politique de la proposition de tarif}

Le Dr Jürg Schlup, président de la FMH, a ouvert la séance en présentant le volet politique de la proposition de tarif actuelle. Selon lui, le TARMED n'est plus à jour et les interventions successives du Conseil fédéral représentent un pas de plus vers un tarif étatique. Les coûts de la santé sont un thème récurrent dans les médias et le débat politique, et il convient donc de se demander si l'on veut des prix fixés au niveau politique ou un tarif approprié et conforme aux règles de l'économie d'entreprise. Dans son volet de mesures visant à freiner les coûts qu'il a récemment mis en consultation, le Conseil fédéral prévoit d'inscrire dans la loi l'obligation de corriger l'augmentation des coûts de l'assurance obligatoire des soins (AOS) par une adaptation annuelle des tarifs. Il veut également inscrire dans

la loi l'institution, par les partenaires tarifaires, d'une organisation nationale chargée du suivi des tarifs ambulatoires, avec la possibilité de l'instituer lui-même si ces derniers ne parviennent pas à s'entendre. Par

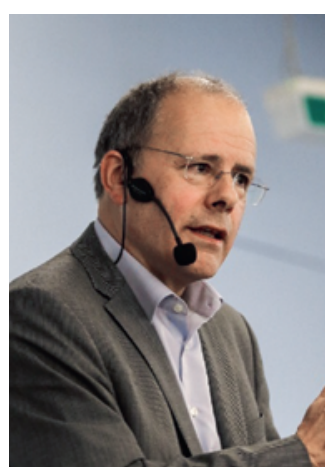

Le Dr Jürg Schlup présente le volet politique de la proposition de tarif. conséquent, si le corps médical ne propose pas maintenant une structure tarifaire révisée, l'autonomie tarifaire risque d'être définitivement perdue. Pour Jürg Schlup, la FMH est tout à fait en mesure de présenter quelque chose. Après des années de travaux intensifs et de négociations, elle a obtenu une structure des prestations dont elle peut être fière. 


\section{Dernière ligne droite pour la révision tarifaire?}

Le Dr Urs Stoffel, membre du Comité central de la FMH, a commencé par rappeler que l'Assemblée des délégués et la Chambre médicale de la FMH ont validé à l'unanimité ce printemps la structure des prestations élaborée dans le cadre du projet TARCO. Pendant près d'une année, pas moins de 150 personnes réparties dans 40 groupes de travail ont révisé l'ensemble des chapitres

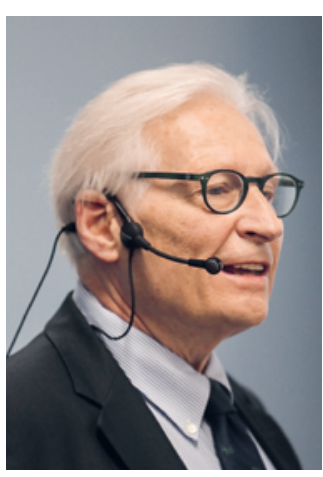

Le Dr Urs Stoffel présente son exposé intitulé «Dernière ligne droite pour la révision tarifaire?". de la structure tarifaire. Le résultat de ce projet a fait entièrement partie des négociations avec les partenaires tarifaires au sein de la société anonyme atstms. Ces derniers mois, les négociations ont en effet notamment porté sur l'intégration du projet TARCO dans la nouvelle structure des prestations commune. Les travaux se sont achevés fin septembre 2018; cette structure des prestations peut donc être présentée cet automne aux organes de la FMH pour décision, ce qui marquera une étape importante.

\section{Consultation sur le volet de mesures de la Confédération visant à freiner les coûts}

Patrick Müller a donné des informations sur la consultation (en cours) des mesures de la Confédération visant à freiner les coûts. Fin 2016, le Département fédéral de l'intérieur (DFI) a convoqué un groupe d'experts internationaux chargé de proposer des mesures pouvant être mises en œuvre rapidement pour freiner les coûts de la santé. Ces mesures seront appliquées en deux volets, conformément à la décision du Conseil fédéral. Le premier volet est en consultation depuis la mi-septembre 2018. Patrick Müller a ensuite présenté les différentes mesures plus en détail. Le Conseil fédéral aimerait inscrire dans la loi l'institution d'une organisation tarifaire nationale pour le secteur ambulatoire, sur le modèle de SwissDRG SA dans le secteur hospitalier (art. 47a LAMal, nouveau). A cet effet, il peut édicter des principes relatifs à la forme et à l'exploitation de cette organisation. Les fournisseurs de prestations sont par ailleurs tenus de livrer à l'organisation les données nécessaires concernant les coûts et les prestations (art. 47b LAMal, nouveau). La consultation de l'OFSP est en cours jusqu'au 14 décembre 2018.

\section{Monitorage de l'intervention du Conseil fédéral dans le TARMED}

Patrick Müller a signalé que, lors de la deuxième intervention tarifaire, le Conseil fédéral a également imposé aux partenaires tarifaires un monitorage détaillé

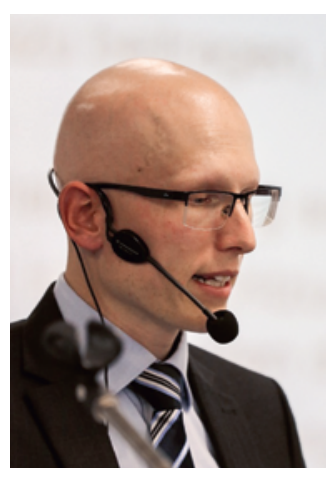

Patrick Müller aborde en détail la consultation des mesures de la Confédération visant à freiner les coûts et le monitorage de la deuxième intervention tarifaire. incluant une livraison gratuite des données à l'OFSP. Les premières données ont été livrées en juillet 2018 et portent sur le premier trimestre 2018. La FMH a décliné toute responsabilité concernant ces données en raison de leur instabilité partielle qui ne permet pas de tirer des conclusions sur la facturation ni sur l'application de la structure tarifaire, en particulier en ce qui concerne les nouvelles limitations. En raison de la complexité des changements, un nombre particulièrement élevé de factures a été annulé durant le premier trimestre, ce qui peut avoir un impact sur les données. Selon la technologie utilisée, les factures annulées ne peuvent pas être supprimées du fichier livré et peuvent, si elles sont nombreuses, fausser la vue d'ensemble. Des chiffres plus fiables ne sont donc pas attendus avant le deuxième trimestre 2018 avec un délai d'au moins 3-4 mois pour la collecte. Dans un rapport de monitorage, la FMH a signalé à l'OFSP les différents problèmes rencontrés suite à la deuxième intervention tarifaire. Une nouvelle livraison est prévue d'ici fin 2018 et permettra une première analyse approfondie.

\section{Informations concernant le domaine du laboratoire}

La Dresse Susanne Christen a annoncé que la motion 17.3969 «Négociation des tarifs des analyses de laboratoire par les partenaires tarifaires" a été acceptée par le Conseil national le 19 septembre 2018, après avoir été acceptée par le Conseil des Etats, et que la liste des analyses devrait ainsi passer d'un tarif officiel imposé à un tarif négocié par les partenaires tarifaires. Elle a ensuite présenté les modifications de la liste des analyses entrées en vigueur au $1^{\mathrm{er}}$ septembre 2018, et en particulier la dénomination de la troponine dans la liste des analyses rapides (position 1734.01); la troponine, test rapide (position 1735.00), a bien été supprimée de la 
liste des analyses complémentaires, mais elle est encore admise lors de consultation à domicile. Elle a par ailleurs expliqué que certains confrères font valoir leurs droits acquis pour facturer des analyses de la liste élargie réservées à des titres de formation postgraduée précis, et reçoivent ensuite à juste titre des demandes de remboursement de la part de tarifsuisse. Il faut

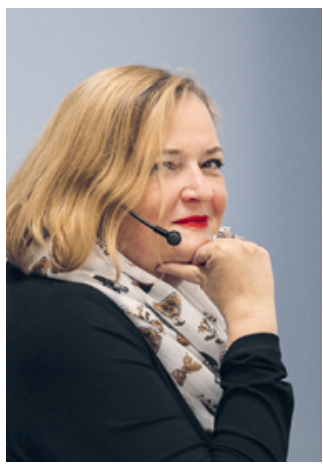

La Dresse Susanne Christen a présenté I'actualité du domaine du laboratoire au cabinet. savoir que la liste des analyses est un tarif officiel, fixé par les autorités, et qu'à ce titre, il n'admet aucune possibilité de droits acquis; ces derniers concernent exclusivement le TARMED. La Dresse Susanne Christen a terminé son exposé par quelques précisions sur l'attestation de formation complémentaire pour la pratique du laboratoire au cabinet médical. Cette attestation fait partie du programme d'assurance-qualité pour le laboratoire de cabinet médical. La participation régulière aux contrôles de qualité externes correspond à une recertification. Pour s'en assurer, tarifsuisse a commencé à exiger de certains médecins la présentation du certificat de participation établi par les centres de contrôle de la qualité durant ces cinq dernières années. Conformément à l'accord passé avec santésuisse dans le cadre de la QUALAB, la participation aux contrôles de qualité externes est enregistrée auprès de SASIS SA à titre d'information à l'intention des assureurs. Par conséquent, nous recommandons aux médecins concernés d'informer les assureurs et/ou tarifsuisse qu'il leur faut

s'adresser à SASIS SA.
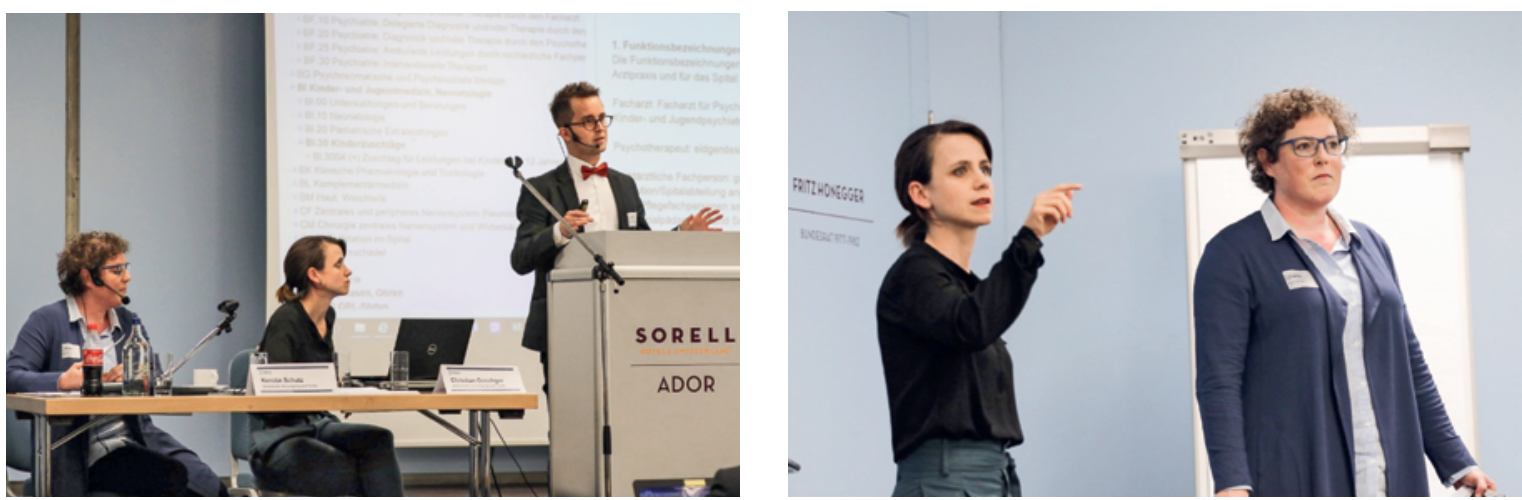

Christian Oeschger, Kerstin Schutz et Sabine Zehnder à propos de la révision du tarif ambulatoire par les partenaires tarifaires.

\section{Révision du tarif ambulatoire par les partenaires tarifaires}

Kerstin Schutz, Sabine Zehnder et Christian Oeschger ont présenté aux délégués les principaux changements dans la structure des prestations partenariale. En plus de la mise à jour des modèles de coûts, certains changements concernent également la nomenclature. Les responsables des groupes de travail informeront les délégués tarifaires des sociétés de discipline médicale plus en détail concernant les changements dans leurs chapitres respectifs et leur fourniront des exemples concrets (paniers de prestations) ces prochains jours. Il est important d'analyser la structure tarifaire révisée sur la base de ces regroupements et de ne pas comparer position par position. La FMH publiera en novembre un article dans le $B M S$ pour informer en détail à ce sujet et présenter les prochaines étapes.

\section{Rétrospective et conclusion}

Le Dr Urs Stoffel a clos la séance en expliquant que la pression politique sur le corps médical continuera d'augmenter et que les coûts de la santé seront le thème de campagne de certains partis pour les élections fédérales 2019. Il a ensuite invité les délégués à saisir l'occasion qui se présente aujourd'hui pour montrer que le corps médical est tout à fait capable de négocier avec ses partenaires tarifaires pour obtenir un tarif révisé, approprié et conforme aux règles de l'économie d'entreprise.

Diapositives: les documents de séance et la présentation PowerPoint sont disponibles sur le site internet de la FMH: www. $\mathrm{fmh}$.ch $\rightarrow$ Tarifs ambulatoires $\rightarrow$ Journée des délégués tarifaires $\rightarrow 27$ septembre $2018 \rightarrow$ Présentation

Prochain rendez-vous: la prochaine Journée des délégués tarifaires aura lieu à l'Hôtel Ador à Berne le 11 avril 2019. Notez d'ores et déjà cette date dans votre agenda!
Correspondance:

FMH

Division Médecine et

tarifs ambulatoires

Baslerstrasse 47

CH-4600 Olten

Tél. 0313591230

Fax 0313591238

tarife.ambulant[at]fmh.ch 